\title{
The DNA damage response pathway in normal hematopoiesis and malignancies
}

\author{
Domenico Delia $^{1} \cdot$ Shuki Mizutani ${ }^{2}$
}

Received: 29 June 2017 / Accepted: 5 July 2017 / Published online: 13 July 2017

(C) The Japanese Society of Hematology 2017

\begin{abstract}
In mammalian cells, the DNA damage response (DDR) prevents the replication and propagation of DNA errors to the next generation, thus maintaining genomic stability. At the heart of the DDR are the related signaling kinases ATM, ATR, and DNA-PK, which regulate DNA repair and associated events such as cell cycle checkpoints, chromatin remodeling, transcription, and ultimately apoptosis. Several findings highlight the occurrence of DDR in hemopoietic stem cells (HSCs), and persistence of DNA lesions in these cells promotes their functional decline and accumulation of leukemogenic mutations. Besides favoring tumor formation and progression, molecular defects that directly or indirectly inactivate certain DDR pathways can provide a therapeutic opportunity, since a reduced ability to repair DNA lesions renders hemopoietic malignancies vulnerable to genotoxic drugs acting also through synthetic lethal interactions. Here, we discuss the essential role of DDR in HSC maintenance and protection against leukemogenesis, and how acquired DDR dysfunctions or pharmacological agents that block this pathway can be effectively exploited for the treatment of various hematopoietic malignancies.
\end{abstract}

Keywords DNA damage response - DNA repair . Hemopoietic stem cell · Leukemogenesis · ATM kinase . ATR kinase

Domenico Delia

domenico.delia@istitutotumori.mi.it

1 Fondazione IRCCS Istituto Nazionale dei Tumori, Via Amadeo 42, 20133 Milan, Italy

2 Kawasaki North Center for Childhood Developmental Disorder/Tokyo Medical and Dental University, 5-26-1 Katahira, Aso-ku, Kawasaki 215-0003, Japan

\section{Introduction}

The cell's genomic DNA is constantly under threat owing to replication errors, endogenous metabolic by-products such as reactive oxygen species, or exposure to environmental mutagens such as air pollutants, cigarette smoke, and solar ultraviolet radiation [1]. Genotoxic agents induce in cells such as DNA single-strand break (SSB) lesions, which are the most frequent, and double strand breaks (DSBs), which occur less frequently, but are the most dangerous as they can lead to chromosomal rearrangements, amplifications, deletions, and overall genomic instability and cancer.

To prevent the replication of DNA errors and thus maintain genomic stability, eukaryotic cells have evolved a machinery, called DNA damage response (DDR), constituted by a network of biochemical pathways that detect, signal, and repair the DNA lesions, while coordinating these events with transient cell cycle arrest. Where the DNA damage is overwhelming, the DDR can drive cells into apoptosis or senescence, thereby preventing the expansion of mutant and potentially tumorigenic cells. The association of DDR defects with cancer is best attested by the existence of cancer-susceptible patients carrying inherited mutations in genes that inactivate the DDR. Notably, the DDR triggers other molecular events that regulate stem cell homeostasis and aging, and defects in DDR can markedly impact these cellular properties.

At the heart of the DDR are the protein kinases ATM, ATR, and DNA-PK, members of the PIKK (phosphatidylinositol 3-kinase-like kinase) family, which are rapidly activated by different DNA breaks and phosphorylate a multitude of substrates, allowing them to jointly orchestrate DNA repair and cell recovery [2]. Albeit they share many similarities, ATM is responsive to DSBs and plays a role in 
multiple cell cycle checkpoints, whereas ATR is responsive to SSBs arising from stalled replication forks or from UV radiation, and functions in the S-phase checkpoint [3], and while ATM promotes homologous recombination (HR), DNA-PK promotes DSBs religation via non-homologous end joining (NHEJ).

Upon sensing DSBs, ATM undergoes activation by autophosphorylation on Ser1981 [4, 5] and gets recruited to the DNA ends by interaction with the Mre11-Rad50-Nbs1 (MRN) complex, the actual sensor of DSBs. The MRN/ ATM interaction at breaks further enhances ATM's kinase activity, allowing it to phosphorylate several substrates, including the histone H2AX at Ser $139(\gamma \mathrm{H} 2 \mathrm{AX})$, a first step in the recruitment of downstream mediators such as MDC1, 53BP1, and BRCA1 [2] which accumulate as foci in megabase regions around the breaks to facilitate repair [6]. Through CtIP-dependent stimulation of MRE11 endonuclease activity which makes an initial single-strand nick $5^{\prime}$ to the DNA end, and the subsequent exonuclease activity of MRE11, EXD2, and EXO1, DNA end resection occurs, followed by RPA loading onto ssDNA ends and subsequent replacement of RPA by RAD51 to build nucleofilaments structures and link the complementary chromatin strands together. In this context, BRCA1 promotes HR by activating DNA-end resection [7], while 53BP1, a key player in DNA repair and signaling, forms a barrier that prevents excessive resection [8].

It should be noted that the DDR also orchestrates local chromatin remodeling through post-translational modifications of core histones and histone-binding proteins, to facilitate the incoming of repair factors and final restoration of the initial structure $[9,10]$. Moreover, given the tightly nature of heterochromatin, DNA breaks occurring in this region require for their efficient repair the activity of ATM that, by phosphorylating and removing Kap1 from heterochromatin, facilitates chromatin relaxation and recruitment of repair factors [11].

A key downstream target of ATM is the effector checkpoint kinase Chk2 [12], which mediates transient arrest at multiple cell cycle phases to provide time for lesions to be repaired. Following its activation by ATM in response to DNA damage, Chk2 induces the phosphorylation of Cdc25A phosphatase and its subsequent degradation, preventing the dephosphorylation and activation of $\mathrm{Cdk} 2$, hence causing a G1/S checkpoint arrest. Chk2 also phosphorylates $\mathrm{Cdc} 25 \mathrm{C}$, causing its nuclear exclusion and impeding it to dephosphorylate and activate the cyclinB1/ Cdk1 complex, thus enforcing a G2/M checkpoint arrest. By phosphorylating p53, Chk2 promotes the transcriptional activation of $\mathrm{p} 53$ and induction of the CDK inhibitor p2 $1^{\text {waf1 }}$, sustaining the G1/S and G2/M arrest [12].

ATR is mainly involved in the response to singlestranded DNA breaks (SSBs) that arise from stalled replication forks in $\mathrm{S}$ phase or as intermediates of repair DNA lesions. Through its association with the ATR interacting protein (ATRIP), ATR is recruited at sites of RPAcoated SSBs and undergoes autophosphorylation, an essential step for full activation of ATR, mediated by the interaction with TopBP1, Rad17, and 9-1-1 complexes [13]. Active ATR phosphorylates several substrates, including the effector kinase Chk1, activating it. Active Chk1 phosphorylates Cdc25A phosphatase and Treslin, which enforce the $\mathrm{G} 2$ and $\mathrm{S}$ phase arrest and suppression of new origin firing. Additional phosphorylation targets of ATR include RPA, WRN, and MCMs which act locally at stalled replication forks, while others such as FANCI and poln participate in DNA repair [13].

\section{Triggering apoptosis in cases of irreparable DNA damage}

Cells with irreparable DNA damage activate a p53-dependent apoptotic process to avoid the propagation of genomically compromised cells. After DNA damage, ATM phosphorylates p53 at Ser15, disrupting the interaction with MDM2, an E3 ubiquitin protein ligase that normally promote the proteasomal degradation of $\mathrm{p} 53$, allowing the stabilization and transactivation of p53 target genes [14]. This activity of $\mathrm{p} 53$ is further potentiated by the simultaneous degradation of the Mdm2-related protein HDMX subsequent to its phosphorylation by ATM at Ser403 and Chk2 at Ser342 and Ser367 [15, 16]. An additional layer of regulation of the activity of p53 in DDR is provided by Hipk2 kinase, whose phosphorylation by ATM in response to lethal levels of DNA damage leads to Hipk2 accumulation and phosphorylation of p53 at Ser46, a deadly phospho-mark which leads to the induction of pro-apoptotic target genes, such as PUMA, BAX, NOXA, and BID. When DNA damage is less severe, Hipk2 does not affect phosphorylation of p53 at Ser46, indicating that HIPK2 acts as a sensor of the severity of DNA damage and thus determines the fate of cells depending on the amount of DNA damage $[17,18]$.

\section{The essential function of DDR in the maintenance of HSCs}

Due to their long life span, HSCs are prone to accumulation of DNA damage that can compromise hematopoiesis, on one hand by progressively reducing the self-renewal capacity and regenerative potential, hence accelerating HSC functional decline and aging over time, and on the other by initiating leukemogenesis. Several data highlight the occurrence of DDR in HSCs and its essential role in 
the maintenance of HSCs and of hematopoietic homeostasis, particularly those from mice carrying DDR-inactivating mutant alleles (e.g., ATM, Brca1, and FANCA) that in humans are associated with inherited diseases (Fig. 1).

Normal long-term HSCs are in quiescent state within the bone-marrow niche, but in response to stress-inducing stimuli such as viral infections, cytopenias, cytokines (e.g., IFNa, G-CSF, and TPO) or transplantation, they are forced to enter cell cycle and this rapid transition provokes replicative DNA damage, as revealed by comet assays and formation of H2AX, 53BP1, and Rad51 foci [19]. This process is also accompanied by an increased metabolic activity and mitochondrial production of ROS, which actually act as DNA-damaging agents in LT-HSCs exiting dormancy [19]. It should be noted that ROS limit the lifespan of HSCs by inducing the activation of $\mathrm{p} 38$ MAPK and expression of p16 ${ }^{\text {Ink4a }}[20]$. Notably, in the absence of a functional Fanconi anemia repair pathway, LT-HSCs show high rates of bone-marrow failure and cell death in response to stressinducing stimuli and markedly lower repopulating capacity compared to the wild-type cell counterparts [19]. Interestingly, Brcal conditional deletion from embryonic hematopoietic cells determines pancytopenia and total loss of HSCs in the adult mice, while heterozygosity for a Brcal mutant allele results in a modest yet significant decrease in

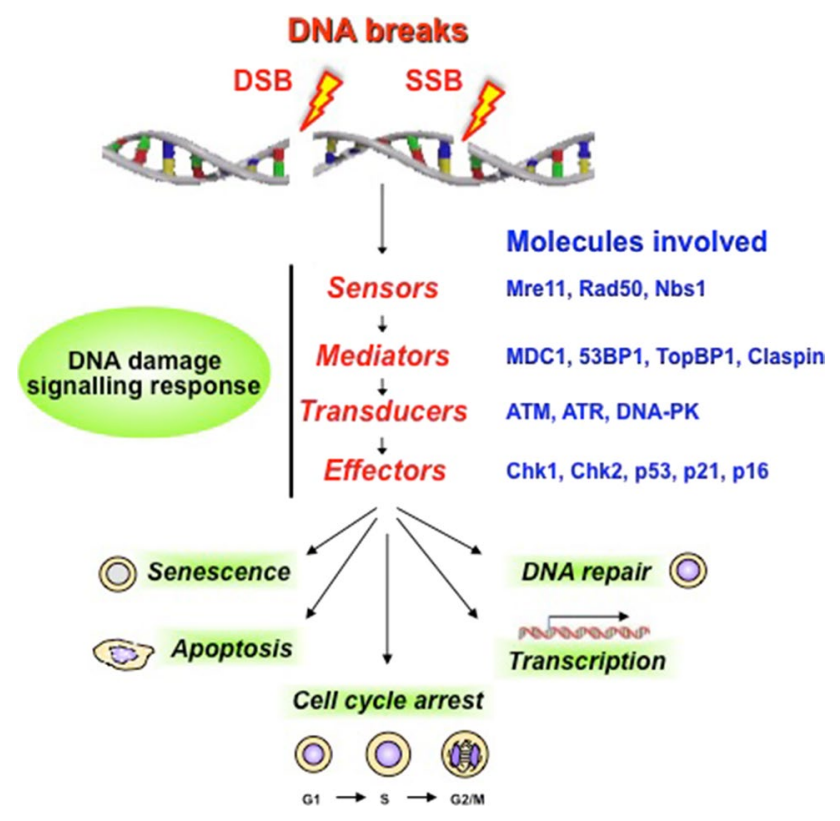

Fig. 1 DDR signaling cascade activated in response to SSBs and DSBs. The cartoon depicts the many molecular components that are recruited in a hierarchical manner at sites of DNA lesions to mediate repair along with other associated events such as transient cell cycle checkpoint arrest, chromatin remodeling, and transcriptional induction. Depending on the cell type and amount of acute or persistent DNA damage, the DDR can drive cells into senescence, or apoptosis via p53 white blood cells, as well as in a deficit in HSC self renewal potential, as assessed by serial bone-marrow transplantation [21], again emphasizing the role of DDR in HSC maintenance.

Studies performed in young and old HSCs have shown an age-dependent accumulation of $\gamma$ - $\mathrm{H} 2 \mathrm{AX}$ (marker of DDR signaling) without any signs of DDR activation or DNA breaks or differences in the expression of DDR proteins [22]. Interestingly, upon cycling old HSCs exhibit, compared to young counterparts, impaired S-phase progression and increased amounts of phosphorylated Chk1 and 53BP1 containing $\gamma$-H2AX foci, indicative of heightened replicative stress, as well as acquisition of chromosomal gaps and breaks, associated with selective downregulation of the MCM DNA helicase components which are essential for DNA replication. It should be noted that another study [23] has shown by single-cell comet assay, a technique that provides a direct measure of DNA breaks, that quiescent HSCs purified from aged mice accumulate to a larger fraction more DNA damage than HSCs from young mice, while conversely, no age-associated differences in accumulated DNA damage are seen in the myeloid or lymphoid progenitors. Moreover, the accumulated DNA damage in the old HSCs is repaired as cells are driven into cycle and many DDR-associated genes are promptly upregulated (e.g., CDKN1a, GADD45, and Rb1). This damage repair in the old HSCs is nevertheless unable to fully restore the hematopoietic function, as evident from their reduced reconstitution capacity and myeloid bias upon transplantation. Thus, the persistence in HSCs of DNA lesions, repairable upon cell cycle re-entry, promotes the functional decline and stepwise accumulation of leukemogenic mutations.

Very recently, it was found that persistent DNA damage signaling in mouse HSCs arising from eroded telomeres induces a myelodysplastic syndrome (MDS) phenotype characterized by blood cytopenia and bone-marrow hypercellularity associated with a myeloid-biased differentiation and tri-lineage dysplasia [24]. At stem cell level, the DNA damage increases the expansion of the LT_HSCs (cKit+/Sca+/Lin-/CD34-/Flk2-) and ST-HSCs (cKit+/ Sca+/Lin-/CD34+/Flk2-), increased frequency of granulocyte-macrophage progenitors, loss of megakaryocyte-erythroid progenitors and decreases in multipotent progenitor cells, and compromises repopulation upon competitive transplantation. Interestingly, the inhibition of ATR but not ATM improves erythroid differentiation, suggesting that mitigating the DNA damage signaling might be useful for the prevention/treatment of MDS. The activation of the DDR characterizes many bone-marrow specimens from MDS patients, according to immunostaining analysis with antibodies anti p-ATM, p-Chk2, and gH2AX, showing highest expression levels especially in patients with 
late refractory anemia with excess blasts (RAEB-1) [25, 26]. This activation of the DDR appears associated with unscheduled DNA replication and activation of ATM, and is not seen in during the progression to overt leukemia, coincident with loss of one or both ATM alleles [26].

\section{The DDR pathway as a therapeutic target and opportunity for hematopoietic malignancies}

Besides favoring tumor formation and progression, DDR defects can provide a therapeutic opportunity, since cancer cells with a reduced ability to repair DNA lesions can acquire hypersensitivity to chemotherapeutic drugs that directly damage the DNA or act through synthetic lethal interactions [27, 28].

In patients with acute myeloid leukemia (AML), activating mutations in the FLT3 tyrosine kinase receptor are frequently observed and associated with poor prognosis, and despite the effectiveness of FLT3 inhibitor-targeted therapies in inducing a high rate of complete remissions, nevertheless, these remissions are not sustained. Recently, Gregory et al. [29] found that inactivation of ATM or its downstream effector G6PD as being synthetic lethal with the FLT3 inhibitor AC220. Interestingly, metabolomic analysis performed on AML cells in vitro showed that while the FLT3 inhibitor impairs the glutathione metabolism and induces mitochondrial oxidative stress, these toxic effects are markedly exacerbated upon ATM or G6PD knockdown. While these findings underscore the role played by FLT3 and ATM in the regulation of metabolic pathways, they also provide evidence supporting a combinatorial therapeutic strategy in FLT3-mutated AML based on chemical inhibition of both FLT3 and ATM.

AML cells with oncogenic rearrangements in MLL are particularly resistant to the conventional treatment with genotoxic drugs, since they have a poor p53 signaling and ensuing p53-dependent cell death. Interestingly, however, ATR inhibition kills cancer cells, even those p53-deficient, by inducing accumulation of replication stress and premature mitotic entry from $\mathrm{G} 2$ phase, both being independent of p53. This evidence and the fact that Chk1, the downstream effector target of ATR, is overexpressed in many hematopoietic malignancies [30] and its abundance in AML negatively correlates with prognosis [31], has prompted investigations on the antitumor activity of ATR inhibition in AML-MLL. Notably, the ATR inhibitor AZ20 shows potent cytotoxic activity against AML-MLL cells in vitro, associated with activation of the DDR, increased replicative damage, and death independent of p53. Moreover, in vivo studies in mice performed with allografts of murine AML-MLL and xenografts of human AML cell line show that the ATR inhibitor, administered as monotherapy, markedly prolongs survival of mice and decreases tumor volume upon treatment with the ATR inhibitor [30]. Likewise, these in vivo studies with AML-MLL demonstrate that inhibition of ATM has a similar therapeutic activity [31].

Significantly, the ATR inhibitor AZ20 or AZD6738 has been shown to exert a synergistic antileukemic activity in vitro in combination with cytarabine in AML cell lines and primary patient samples [32]. Specifically, inhibition of ATR in these cells induces DNA replication stress, abrogation of the G2/M cell cycle checkpoint, and downregulation of the ribonucleotide reductase M1 and M2 subunits, further increasing genotoxic damage and potentiating the cytotoxic activity of cytarabine. Thus, the combination of ATR inhibitors with cytarabine might provide a powerful opportunity for the treatment of AML.

Notwithstanding the widely recognized role of DDR as an anticancer barrier by way of its capacity to prevent oncogene-induced replication stress and malignant transformation of precancerous lesions [33], recent findings with bone-marrow myeloid cells expressing the MLLAF9 fusion oncogene, whose leukemogenic effect reflects its capacity to sustain a differentiation block, show that inhibition of the DDR pathway components ATM, ATR, or Brca1 promotes loss of MLL-AF9 blasts and terminal differentiation, strongly supporting the hypothesis that in some developing malignancies, the DDR might act as an oncogenic driver $[34,35]$. In addition, these findings suggest that DDR inhibitors may act as differentiation therapy for the treatment of certain myeloid leukemias.

Chk1 overexpression, both at transcriptional and protein level, besides being an independent prognostic marker associated with increased risk of relapse and poor survival in AML patients previously treated with cytarabine-based chemotherapy, provides a new target to overcome resistance. Indeed, its has been shown that Chk1 over-expression in these leukemias promotes proliferation and viability by enhancing both clonogenicity and DNA replication fork progression in response to cytarabine, and in agreement with this, chemical inhibition of Chk1 by SCH900776 represses colony formation and replication fork progression, and simultaneously hypersensitizes cells to cytarabine to the same extent as those expressing low Chk1 levels [31]. Hence, in AML patients overexpressing Chk1, this kinase not only ensures the maintenance of replication forks upon treatment with cytarabine, and it also provides a viable target for therapeutic intervention.

Deregulated expression of Chk1 is also found in primary leukemic blasts from B-ALL patients [36]. When treated in vitro with prexasertib, a pharmacologic inhibitor of Chk1, these blasts are selectively killed, whereas normal mononuclear cells are spared [37]. Moreover, studies with primary B- and T-ALL have shown that prexasertib 
markedly potentiates the cytotoxic activity of clofarabine, a purine nucleoside analogue commonly used for the treatment of ALL patients [38].

Chk1 has also been found deregulated and hyperactive (as determined by its phosphorylation on Serine 296) in a subgroup of patients with proliferative T-ALL, likely due to the excessive replication stress and deregulated S-phase progression, resulting in high levels of the replication stress and DNA damage markers phospho-RPA32 and $\gamma \mathrm{H} 2 \mathrm{AX}$. In these lymphoblastic leukemias, Chk1 is indispensable for cell survival [39], since its inhibition in vitro with the small-molecule inhibitor PF-00477736 culminates in the accumulation of DNA replication intermediates, activation of the ATM/Chk2-dependent DDR, and caspase-3-dependent apoptosis [39]. Furthermore, this anti-leukemic effect is also observed in vivo in mice xenotransplantated with human T-ALL, where systemic administration of PF-00477736 limits tumor growth [39]. Altogether, these observations lend support to clinical trials against the ATRChk1 pathway in T-ALL patients exhibiting hyperactive Chk1 [40].

Diffuse large B cell lymphoma (DLBCL) is one of the most common lymphoma subtypes, characterized by aggressive clinical course associated, in a subset of cases, with high genomic instability, somatic and germline mutations in DDR genes, including mutations in mismatch repair genes (EXO1, MSH2, and MSH6), NHEJ (Artemis, DNA-PKcs, Ku70, and Ku80), CHEK2, and PARP1 [41]. More recently, an association in DLBCL between the frequently overexpressed c-myc oncogene, DNA replication stress, and constitutive activation of the DDR has been found. Moreover, patients with DLBCL being positive for $\gamma \mathrm{H} 2 \mathrm{AX}$, a biomarker of DNA damage, exhibit a poor overall survival following treatment with R-CHOP compared with those negative for $\gamma \mathrm{H} 2 \mathrm{AX}$. Notably, the inhibition of Chk1 with AZD-7762 or PF-0477736 induces the accumulation of DNA damage and apoptosis of DLBCL cells, providing support for therapeutic intervention with Chk1 inhibitors [42].

Chronic lymphocytic leukemia (CLL) is an extremely heterogeneous disease in its clinical manifestations and treatment response. Up to $80 \%$ of CLL patients present cytogenetic abnormalities which in $25 \%$ of cases involve deletion 11q22-q23 (del(11q)), the locus of the ATM gene and in $5-8 \%$ of cases deletion $17 \mathrm{p} 13(\operatorname{del}(17 \mathrm{p}))$, the locus of the p53 tumor suppressor gene. Notably, $40 \%$ of patients with del(11q) show inactivating mutations of the second ATM allele. These CLL subtypes are both characterized by resistance to genotoxic chemotherapies and short overall survival.

Recent in vitro studies have shown that pharmacologic inhibition of ATR in p53- or ATM-defective CLL cells selectively induces replication fork stalls, accumulation of unrepaired DNA damage, and mitotic cell death [43, 44]. This synthetic lethal effect is also observed in xenograft models of p53- or ATM-defective primary CLL, where AZD6738 treatment not only significantly reduces tumor load [44], but also overcomes resistance to chemotherapeutics used in the first-line treatment of CLL such as chlorambucil, fludarabine, or bendamustine. Collectively, these results support the combined use of ATR inhibitor with a range of existing therapeutic agents for CLL.

PARP1 is an enzyme of the DDR pathway with a key role in detecting and binding to SSBs, initiating the repair of these lesions. The inhibition of PARP1 induces the formation of SSBs, which then evolve into DSBs that in normal cells are repaired by the HR [45]. However, in cells where the HR is defective, such as those with mutated BRCA1 or BRCA2, PARP1 inhibition induces an excessive accumulation of genotoxic damage which triggers cell death, and this synthetic lethality effect has been effectively exploited in the treatment of Brca1-defective solid tumors [46]. More recent studies have shown that PARP1 inhibitors may also find application for the treatment of certain hematological malignancies. Indeed, a subset of AML where the genetic defect represses a variety of genes involved in DDR, such as those having the AML1-ETO translocation [47] or in cases where an aberrant transcription network reduces the expression of Brcal in primary AML [48] or relapsed cases [49].

Among the B cell precursor lineage ALL, about $1 \%$ carry the chromosomal translocation that creates the TCF3HLF fusion protein, which confers extremely poor prognosis. Recently, it has been found that TCF3-HLF expression downregulates $\mathrm{MCPH} 1$, a protein involved in DDR and stabilization of Brcal [50], causing a drop in Brcal protein levels, suppression of the HR pathway and elevation of the endogenous DSBs, and markedly increased cytotoxic activity to the PARP inhibitor olaparib, both in vitro and in an in vivo mouse models of human TCF3-HLF leukemia xenograft, especially when olaparib is used in combination with the alkylating agent temozolomide [51].

The evidence that ATM-deficient tumors are hypersensitive to PARP inhibitors has prompted investigations to determine the response of ATM-defective CLL to PARP inhibition. Studies performed with proliferating ATM-deficient CLL show increased sensitivity to the PARP inhibitor olaparib compared with those expressing ATM [52] suggesting a strategy for the treatment of ATM-defective CLL. A phase I/II clinical trial is investigating the use of olaparib in CLL patients stratified by ATM status (11q deletion or ATM mutation: ISRCTN34386131 http://www.isrctn. com/ISRCTN34386131) and phase I studies of talazoparib have been undertaken in hematological malignancies (NCT01399840). 
It should be noted, however, that a recent study, while confirming the cytotoxic activity of the potent PARP inhibitor talazoparib in patient-derived CLL cells, failed to find an association between PARP activity, ATM loss, or levels of oxidative DNA damage [53].

Mutations in isocitrate dehydrogenase-1 (IDH1), the enzyme that catalyzes the conversion of isocitrate to $\alpha$-ketoglutarate, are frequently found in AML with normal karyotype and have an adverse effect on disease-free survival and complete remission rate [54]. Most mutations in IDH1 confer a neomorphic activity on the enzymes, such that they convert $\alpha$-ketoglutarate to (R)-2-hydroxyglutarate, which is considered an oncometabolite having effects on chromatin methylation and cellular differentiation [55]. Mutant IDH1 inhibits TET2, an enzyme involved in cytosine demethylation, resulting in disrupted DNA methylation and differentiation. In AML, mutations in TET2 are mutually exclusive with those in IDH1, and confer clinically distinctive features. Interestingly, IDH1-mutations in mice have a profound effect on HSC homeostasis, inducing a marked drop in the number and self-renewal capacity of LT-HSCs [56]. Notably, mutant IDH1 represses the expression of ATM and activation of the DDR signaling pathway, leading to the accumulation of DNA damage, which in turn impairs the self-renewal capacity of LT-HSCs. The impaired DDR in IDH1-mutated cells confers hypersensitivity to genotoxic agents such as ionizing radiation and daunorubicin, and in line with this, IDH1-AML patients show a better prognosis than TET2-AML patients following daunorubicin treatment [56]. Most recently, the finding that cells harboring mutant IDH1 (and IDH2) markedly repress the repair of DNA damage by homologous recombination has provided a mechanistic evidence explaining the enhanced chemosensitivity and radiosensitivity of IDH1 mutant tumors. Moreover, since HR defects induce synthetic lethal interactions with PARP inhibitors (e.g., olaparib) or ATR inhibitors (e.g., VE-822), studies performed with patient-derived AML cells harboring IDH1 mutations causing HR suppression and DDR defects have shown that these cells are, compared to matched IDH1 wild-type cases, highly vulnerable to PARP inhibitors and ionizing radiation [55]. The vulnerability to PARP inhibition may thus be therapeutically exploited in the case of IDH1-mutant HR-defective AML.

\section{References}

1. Ciccia A, Elledge SJ. The DNA damage response: making it safe to play with knives. Mol Cell. 2010;40:179-204 (Review).

2. Shiloh Y, Ziv Y. The ATM protein kinase: regulating the cellular response to genotoxic stress, and more. Nat Rev Mol Cell Biol. 2013;14:197-210 (Review).
3. Blackford AN, Jackson SP. ATM, ATR, and DNA-PK: the trinity at the heart of the DNA damage response. Mol Cell. 2017;66:801-17 (Review).

4. Bakkenist CJ, Kastan MB. DNA damage activates ATM through intermolecular autophosphorylation and dimer dissociation. Nature. 2003;421:499-506.

5. Goldstein M, Kastan MB. The DNA damage response: implications for tumor responses to radiation and chemotherapy. Annu Rev Med. 2015;66:129-43 (Review).

6. Bekker-Jensen S, Mailand N. Assembly and function of DNA double-strand break repair foci in mammalian cells. DNA Repair (Amst). 2010;9:1219-28 (Review).

7. Schlegel BP, Jodelka FM, Nunez R. BRCA1 promotes induction of ssDNA by ionizing radiation. Cancer Res. 2006;66:5181-9.

8. Isono M, Niimi A, Oike T, Hagiwara Y, Sato H, Sekine R, et al. BRCA1 directs the repair pathway to homologous recombination by promoting 53BP1 dephosphorylation. Cell Rep. 2017;18:520-32.

9. Price BD, D'Andrea AD. Chromatin remodeling at DNA doublestrand breaks. Cell. 2013;152:1344-54.

10. Papamichos-Chronakis M, Peterson CL. Chromatin and the genome integrity network. Nat Rev Genet. 2013;14:62-75 (Review).

11. Goodarzi AA, Jeggo P, Lobrich M. The influence of heterochromatin on DNA double strand break repair: getting the strong, silent type to relax. DNA Repair (Amst). 2010;9:1273-82 (Review).

12. Zannini L, Delia D, Buscemi G. CHK2 kinase in the DNA damage response and beyond. J Mol Cell Biol. 2014;6:442-57 (Review).

13. Yazinski SA, Zou L. Functions, regulation, and therapeutic implications of the ATR checkpoint pathway. Annu Rev Genet. 2016;50:155-73.

14. Cheng Q, Chen J. Mechanism of p53 stabilization by ATM after DNA damage. Cell Cycle. 2010;9:472-8.

15. LeBron C, Chen L, Gilkes DM, Chen J. Regulation of MDMX nuclear import and degradation by Chk2 and 14-3-3. EMBO J. 2006;25(6):1196-206.

16. Pereg Y, Lam S, Teunisse A, Biton S, Meulmeester E, Mittelman L, et al. Differential roles of ATM- and Chk2-mediated phosphorylations of Hdmx in response to DNA damage. Mol Cell Biol. 2006;26(18):6819-31.

17. Kuwano Y, Nishida K, Akaike Y, Kurokawa K, Nishikawa T, Masuda $\mathrm{K}$, et al. Homeodomain-interacting protein kinase-2: a critical regulator of the DNA damage response and the epigenome. Int J Mol Sci. 2016;17:1638.

18. Matt S, Hofmann TG. The DNA damage-induced cell death response: a roadmap to kill cancer cells. Cell Mol Life Sci. 2016;73(15):2829-50.

19. Walter D, Lier A, Geiselhart A, Thalheimer FB, Huntscha S, Sobotta MC, et al. Exit from dormancy provokes DNA-damage-induced attrition in haematopoietic stem cells. Nature. 2015;520(7548):549-52.

20. Ito K, Hirao A, Arai F, Takubo K, Matsuoka S, Miyamoto $\mathrm{K}$, et al. Reactive oxygen species act through p38 MAPK to limit the lifespan of hematopoietic stem cells. Nat Med. 2006;12(4):446-51.

21. Mgbemena VE, Signer RA, Wijayatunge R, Laxson T, Morrison SJ, Ross TS. Distinct Brca1 mutations differentially reduce hematopoietic stem cell function. Cell Rep. 2017;18(4):947-60.

22. Flach J, Bakker ST, Mohrin M, Conroy PC, Pietras EM, Reynaud $\mathrm{D}$, et al. Replication stress is a potent driver of functional decline in ageing haematopoietic stem cells. Nature. 2014;512(7513):198-202.

23. Beerman I, Seita J, Inlay MA, Weissman IL, Rossi DJ. Quiescent hematopoietic stem cells accumulate DNA damage during 
aging that is repaired upon entry into cell cycle. Cell Stem Cell. 2014;15(1):37-50.

24. Colla S, Ong DS, Ogoti Y, Marchesini M, Mistry NA, CliseDwyer K, et al. Telomere dysfunction drives aberrant hematopoietic differentiation and myelodysplastic syndrome. Cancer Cell. 2015;27(5):644-57.

25. Popp HD, Naumann N, Brendel S, Henzler T, Weiss C, Hofmann WK, et al. Increase of DNA damage and alteration of the DNA damage response in myelodysplastic syndromes and acute myeloid leukemias. Leuk Res. 2017;57:112-8.

26. Horibe S, Takagi M, Unno J, Nagasawa M, Morio T, Arai A, et al. DNA damage check points prevent leukemic transformation in myelodysplastic syndrome. Leukemia. 2007;21(10):2195-8.

27. Brown JS, O'Carrigan B, Jackson SP, Yap TA. Targeting DNA repair in cancer: beyond PARP inhibitors. Cancer Discov. 2017;7(1):20-37.

28. Pearl LH, Schierz AC, Ward SE, Al-Lazikani B, Pearl FM. Therapeutic opportunities within the DNA damage response. Nat Rev Cancer. 2015;15(3):166-80.

29. Gregory MA, D’Alessandro A, Alvarez-Calderon F, Kim J, Nemkov T, Adane B, et al. ATM/G6PD-driven redox metabolism promotes FLT3 inhibitor resistance in acute myeloid leukemia. Proc Natl Acad Sci USA. 2016;113(43):E6669-78.

30. Morgado-Palacin I, Day A, Murga M, Lafarga V, Anton ME, Tubbs A, et al. Targeting the kinase activities of ATR and ATM exhibits antitumoral activity in mouse models of MLL-rearranged AML. Sci Signal. 2016;9(445):ra91.

31. David L, Fernandez-Vidal A, Bertoli S, Grgurevic S, Lepage B, Deshaies D, et al. CHK1 as a therapeutic target to bypass chemoresistance in AML. Sci Signal. 2016;9(445):ra90.

32. Ma J, Li X, Su Y, Zhao J, Luedtke DA, Epshteyn V, et al. Mechanisms responsible for the synergistic antileukemic interactions between ATR inhibition and cytarabine in acute myeloid leukemia cells. Sci Rep. 2017;7:41950. doi:10.1038/srep41950.

33. Bartek J, Bartkova J, Lukas J. DNA damage signalling guards against activated oncogenes and tumour progression. Oncogene. 2007;26(56):7773-9 (Review).

34. Santos MA, Faryabi RB, Ergen AV, Day AM, Malhowski A, Canela A, et al. DNA-damage-induced differentiation of leukaemic cells as an anti-cancer barrier. Nature. 2014;514(7520):107-11.

35. Weiss CN, Ito K. DNA damage: a sensible mediator of the differentiation decision in hematopoietic stem cells and in leukemia. Int J Mol Sci. 2015;16(3):6183-201.

36. Di Rora' AG, Iacobucci I, Martinelli G. The cell cycle checkpoint inhibitors in the treatment of leukemias. J Hematol Oncol. 2017;10(1):77.

37. Di Rorà AG, Iacobucci I, Imbrogno E, Papayannidis C, Derenzini E, Ferrari A, et al. Prexasertib, a Chk1/Chk2 inhibitor, increases the effectiveness of conventional therapy in B-/Tcell progenitor acute lymphoblastic leukemia. Oncotarget. 2016;7(33):53377-91.

38. Iacobucci I, Di Rorà AG, Falzacappa MV, Agostinelli C, Derenzini E, Ferrari A, et al. In vitro and in vivo single-agent efficacy of checkpoint kinase inhibition in acute lymphoblastic leukemia. J Hematol Oncol. 2015;8:125. doi:10.1186/s13045-015-0206-5.

39. Sarmento LM, Póvoa V, Nascimento R, Real G, Antunes I, Martins LR, et al. CHK1 overexpression in T-cell acute lymphoblastic leukemia is essential for proliferation and survival by preventing excessive replication stress. Oncogene. 2015;34(23):2978-90.

40. Sarmento LM, Barata JT. CHK1 and replicative stress in T-cell leukemia: can an irreverent tumor suppressor end up playing the oncogene? Adv Biol Regul. 2016;60:115-21 (Review).
41. de Miranda NF, Peng R, Georgiou K, Wu C, Falk Sörqvist $\mathrm{E}$, Berglund $\mathrm{M}$, et al. DNA repair genes are selectively mutated in diffuse large B cell lymphomas. J Exp Med. 2013;210(9):1729-42.

42. Derenzini E, Agostinelli C, Imbrogno E, Iacobucci I, Casadei B, Brighenti E, et al. Constitutive activation of the DNA damage response pathway as a novel therapeutic target in diffuse large B-cell lymphoma. Oncotarget. 2015;6(9):6553-69.

43. Kwok M, Davies N, Agathanggelou A, Smith E, Petermann E, Yates E, et al. Synthetic lethality in chronic lymphocytic leukaemia with DNA damage response defects by targeting the ATR pathway. Lancet. 2015;385(Suppl 1):S58.

44. Kwok M, Davies N, Agathanggelou A, Smith E, Oldreive C, Petermann E, et al. ATR inhibition induces synthetic lethality and overcomes chemoresistance in TP53-or ATM-defective chronic lymphocytic leukemia cells. Blood. 2016;127(5):582-95.

45. Pommier Y, O'Connor MJ, de Bono J. Laying a trap to kill cancer cells: PARP inhibitors and their mechanisms of action. Sci Transl Med. 2016;8(362):362ps17 (Review).

46. Zhao L, So CW. PARP-inhibitor-induced synthetic lethality for acute myeloid leukemia treatment. Exp Hematol. 2016;44(10):902-7.

47. Alcalay M, Meani N, Gelmetti V, Fantozzi A, Fagioli M, Orleth A, et al. Acute myeloid leukemia fusion proteins deregulate genes involved in stem cell maintenance and DNA repair. J Clin Invest. 2003;112(11):1751-61.

48. Faraoni I, Compagnone M, Lavorgna S, Angelini DF, Cencioni MT, Piras E, et al. BRCA1, PARP1 and $\gamma \mathrm{H} 2 \mathrm{AX}$ in acute myeloid leukemia: role as biomarkers of response to the PARP inhibitor olaparib. Biochim Biophys Acta. 2015;1852(3):462-72.

49. Moskwa P, Buffa FM, Pan Y, Panchakshari R, Gottipati P, Muschel RJ, et al. miR-182-mediated downregulation of BRCA1 impacts DNA repair and sensitivity to PARP inhibitors. Mol Cell. 2011;41(2):210-20.

50. Lin SY, Rai R, Li K, Xu ZX, Elledge SJ. BRIT1/MCPH1 is a DNA damage responsive protein that regulates the Brca1-Chk1 pathway, implicating checkpoint dysfunction in microcephaly. Proc Natl Acad Sci USA. 2005;102(42):15105-9.

51. Piao J, Takai S, Kamiya T, Inukai T, Sugita K, Ohyashiki K, et al. Poly (ADP-ribose) polymerase inhibitors selectively induce cytotoxicity in TCF3-HLF-positive leukemic cells. Cancer Lett. 2017;386:131-40.

52. Weston VJ, Oldreive CE, Skowronska A, Oscier DG, Pratt G, Dyer MJ, et al. The PARP inhibitor olaparib induces significant killing of ATM-deficient lymphoid tumor cells in vitro and in vivo. Blood. 2010;116(22):4578-87.

53. Herriott A, Tudhope SJ, Junge G, Rodrigues N, Patterson MJ, Woodhouse L, et al. PARP1 expression, activity and ex vivo sensitivity to the PARP inhibitor, talazoparib (BMN 673), in chronic lymphocytic leukaemia. Oncotarget. 2015;6(41):43978-91.

54. Virijevic M, Karan-Djurasevic T, Marjanovic I, Tosic N, Mitrovic M, Djunic I, et al. Somatic mutations of isocitrate dehydrogenases 1 and 2 are prognostic and follow-up markers in patients with acute myeloid leukaemia with normal karyotype. Radiol Oncol. 2016;50(4):385-93.

55. Sulkowski PL, Corso CD, Robinson ND, Scanlon SE, Purshouse $\mathrm{KR}$, Bai $\mathrm{H}$, et al. 2-Hydroxyglutarate produced by neomorphic IDH mutations suppresses homologous recombination and induces PARP inhibitor sensitivity. Sci Transl Med. 2017;9(375).

56. Inoue $\mathrm{S}, \mathrm{Li} \mathrm{WY}$, Tseng $\mathrm{A}$, Beerman I, Elia AJ, Bendall SC, et al. Mutant IDH1 downregulates ATM and alters DNA repair and sensitivity to DNA damage independent of TET2. Cancer Cell. 2016;30:337-48. 